Letter to the Editor

\title{
Performance of QuantiFERON TB in a student population at low risk of tuberculosis
}

\author{
Paola Molicotti, Alessandra Bua, Stefania Zanetti \\ Department of Biomedical Science, University of Sassari, Sassari, Italy
}

Key words: tuberculosis; Quantiferon; M. tuberculosis

J Infect Dev Ctries 2012; 6(1):100-101.

(Received 02 November 2011 - Accepted 17 November 2011)

Copyright (C) 2012 Molicotti et al. This is an open-access article distributed under the Creative Commons Attribution License, which permits unrestricted use, distribution, and reproduction in any medium, provided the original work is properly cited.

\section{Introduction}

Every year throughout the world, eight million new cases of tuberculosis (TB) are identified, and most of these individuals are infected with Mycobacterium tuberculosis (MTB) [1]. One third of the world's population is infected with MTB and they constitute a reservoir of infection. Eighty percent of all cases worldwide occur in 22 highburden, mainly resource-poor countries [2]. The devastating impact of tuberculosis on vulnerable populations is also driven by its deadly synergy with HIV [3]. Therefore, building capacity and enhancing universal access to rapid and accurate laboratory diagnostics are necessary to control TB and HIV-TB co-infection in resource-limited countries.

The tuberculin skin test (TST) was, until recently, the only tool for diagnosing latent tuberculosis infection (LTBI) [4]. It has several limitations, such as low specificity, which may lead to false positive reactions as consequence of crossreactivity with BCG vaccine or in individuals who have come in contact with environmental nonpathogenic mycobacteria [4]. An effective screening program of LTBI therefore requires an alternative diagnostic approach that has greater specificity and correctly identifies individuals infected with MTB. The use of the QuantiFERON TB Gold (QFT) test to detect MTB was approved in 2005 by the US Food and Drug Administration. It identifies the IFN- $\gamma$ produced from antigen Esat- 6 and CFP-10 encoded by the RD1 region specific for the TB complex. The latest version of QFT, called QuantiFERON-TB Gold In-Tube (QFT-GIT) also includes TB 7.7 antigen [5]. Recently in Northern Sardinia, Italy, several cases of TB were detected in working and school communities. In 2008, for example, there was a microepidemic of $\mathrm{TB}$ in a primary school [6], while in 2009 another outbreak was reported in a secondary school (data not published). In view of these previous outbreaks of $\mathrm{TB}$, and because screening measures have not been conducted in Italian schools for many years, the purpose of this investigation was to perform an epidemiological study using the QFT immunoassay in a university student population to identify possible cases and thereby control the spread of LTBI.

\section{Methodology}

This study, which was approved by the ethics committees of the Public Health Service of Sassari, involved 80 students enrolled in the Faculty of Pharmacy, University of Sassari. After receiving written consent from the study participants, $5 \mathrm{ml}$ of venous blood were collected from each subject. All blood samples were drawn at the office of Public Hygiene of the Local Health Service of Sassari and tested with QFT (Cellestis, Victoria, Australia) at the mycobacteriology laboratory of the University of Sassari. The immunoassay was performed according to the manufacturer's instructions included in the kit, and each response with a cut-off of IFN- $\gamma \geq 0.35 \mathrm{IU} / \mathrm{ml}$ was scored positive.

\section{Results}

Sixty female and 20 male students aged between 21 and 24 years were included in the study. None of them reported having had contact with patients with active TB. None of the students was tested with TST assay. QFT was negative in all subjects tested.

\section{Discussion}

In Italy screening for tuberculosis in community settings such as school and work has 
not been performed since the early 1980 s. Considering that in these settings there are conditions of crowding and age-related susceptibility that favour the emergence of tuberculosis outbreaks [7], there is an increased risk that the disease may spread more easily. In this work we performed an epidemiological surveillance in a student population at low risk of TB. The health survey was conducted using the QFT immunological assay, and results obtained indicated the absence of tuberculosis infection in the population examined.

We did not perform the TST in this study to avoid its several limitations, such as the return visit for reading the results in the subjects tested.

This type of surveillance would be very important in those countries where TB is endemic, particularly in the developing world. In these countries BCG vaccine coverage is high. In South Africa, for example, its coverage is over 95\% [8]. Consequently, the greater specificity (especially in BCG-vaccinated populations) and sensitivity of the QFT assay compared to those of the TST make its use more advantageous for TB surveillance. In these developing countries, which are high-burden settings, it could be interesting to determine the predictive value for subsequent TB disease using QFT compared to TST in adolescents who are currently under investigation as a target group for TB vaccines. Furthermore, the predictive value of interferon gamma release assays (IGRA) for the subsequent onset of TB disease will be helpful in planning clinical trials of novel TB vaccines.

Economics have been an important determinant in the use of QFT in developing countries. Although IGRA is more expensive than TST, health economic analyses have found that it is costeffective as it reduces the number of individuals needing unnecessary chemoprophylaxis. The study that we conducted was important because the identification of individuals with latent $\mathrm{TB}$ is a major benefit to public health as it involves a significant reduction not only of individual risk, but it also helps to reduce the reservoir of infected individuals, which represent the primary source for the potential development of new cases of active $\mathrm{TB}$ in the future.

\section{References}

1. World Health Organization. Global tuberculosis control 2010 (2010) In: Stop TB Department, ed. Geneva: WHO. http://whqlibdoc.who.int/publications/2010/97892415640 669 eng.pdf.

2. World Health Organization, Department of HIV/AIDS, Stop TB Department (2011) Guidelines for intensified tuberculosis case finding and isoniazid preventive therapy for people living with HIV in resource constrained settings.whqlibdoc.who.int/publications/2011/978924150 0708eng/pdf.

3. Delogu G and Fadda G (2009) The quest for a new vaccine against tuberculosis. $\mathrm{J}$ Infect Developing Countries 3:5-15.

4. Woldehanna S and Volmink J (2004) Treatment of latent tuberculosis infection in HIV infected persons. Cochrane Database Syst Rev 1: CD000171.

5. Diel R, Loddenkemper R, Nienhaus A (2009) Evidencebased comparison of commercial interferon-gamma release assays for detecting active tuberculosis: a metaanalysis. Chest 137: 952-968.

6. Molicotti P, Bua A, Mela G, Olmeo P, Delogu R, Ortu S, Sechi LA, Zanetti S (2008) Performance of QuantiFERON-TB testing in a tuberculosis outbreak at a primary school. J Pediatr 152: 585-586. PubMed PMID: 18346520.

7. Sacks JJ, Brenner ER, Breeden DC, Anders HM, Parker RL (1985) Epidemiology of tuberculosis outbreak in a South Carolina junior high school. Am J Public Health 75: 361-365.

8. Farhat M, Greenaway C, Pai M, Menzies D (2006) Falsepositive tuberculin skin tests: what is the absolute effect of BCG and non-tuberculous mycobacteria? Int J Tuberc Lung Dis 10: 1192-1204.

\section{Corresponding author}

Alessandra Bua

Department of Biomedical Science

University of Sassari

Viale San Pietro 43b

07100 Sassari, Italy

Telephone: +39079229807 ; Fax: +39079212345

Email: ale.b76@email.it

Conflict of interests: No conflict of interests is declared. 